\title{
Neuroanatomical basis of behavioral disturbances in patients with prefrontal lesions
}

\author{
ANDREA SLACHEVSKY ${ }^{1,2}$, MARCELA PEÑA ${ }^{3,4}$, CAROLINA PÉREZ $^{2}$, EDUARDO \\ BRAVO $^{5}$ and PATRICIA ALEGRÍA ${ }^{2}$
}

\author{
${ }^{1}$ Programme of Molecular and Clinical Pharmacology, ICBM and Department of Neurological Sciences, \\ School of Medicine, Universidad de Chile, Santiago, Chile. \\ ${ }^{2}$ Department of Neurology, El Salvador Hospital, Santiago, Chile. \\ ${ }^{3}$ Department of Psychology, Pontificia Universidad Católica de Chile, Santiago, Chile. \\ ${ }^{4}$ International School for Advanced Studies (SISA), Trieste, Italy. \\ 5 Asenjo Institute of Neurosurgery, Chile.
}

\begin{abstract}
The role of the frontal lobe in control of behavioral and cognitive abilities is explored in a group of 34 patients with brain lesions restricted to the prefrontal cortex. The scores in both structured behavioral questionnaires and standard neuropsychological tests were analyzed using the injured area of the frontal lobe as the independent variable. Our results show that patients with simultaneous lesions in supero- and inferomedial areas of the prefrontal cortex exhibit higher behavioral disturbances. Bilateral lesions also are associated with greater behavioral troubles. On the contrary, cognitive abilities are globally impaired in prefrontal patients. Results are discussed in relation to current models of the organization of the prefrontal cortex and its role on behavior control.
\end{abstract}

Key terms: dysexecutive syndrome, prefrontal lesions, behavior.

\section{INTRODUCTION}

The case of Phineas Gage, the first welldescribed patient with a prefrontal lesion, revealed that prefrontal lesions can cause severe trouble in everyday life and a profound disruption of personality (MacMillan, 1996). Damage to the prefrontal cortex may lead to a set of symptoms collectively known as "dysexecutive syndrome," characterized by changes in emotion, personality, motivation, and behavior as well as in cognitive aspects of the mind (Chan, 2001). The development of experimental neuropsychology has allowed to identify and to document numerous taskspecific cognitive deficits in patients with prefrontal lesions, such as impairments in response initiation, response suppression, focused attention, rule deduction, ruling maintenance, set-shifting, problem solving, and planning (Godefroy, 2003). Nevertheless, single-case studies of prefrontal patients, such as EVR described by Eslinger and Damasio (1985), have revealed that patients with prefrontal lesions can perform quite well in a series of cognitive tasks despite serious troubles in social behavior. Indeed, some authors have proposed the existence of a modular organization of the frontal cortex in human, with cognitive functions being impaired after damage to the dorsolateral prefrontal cortex and social skills being disturbed when the ventromedial prefrontal areas are affected (Sarazin et al., 1998). The above-mentioned cases revealed that, besides the study of cognitive state using traditional tasks, the analysis of behavior in daily life is crucial for understanding the role of prefrontal cortex in behavior control.

Corresponding author: Andrea Slachevsky, Programme of Molecular and Clinical Pharmacology, ICBM, School of Medicine, Universidad de Chile, Avda. Independencia 1027, Santiago, Chile, Tel: (56-2) 978-6050, Fax: (56-2) 737-2783, E-mail: aslachevsky@adsl.tie.cl 
The differences described between cognitive abilities and daily life behavior in prefrontal patients could be related to methodological as well as neurobiological/ cognitive factors (Shallice and Burgess, 1991).

From a methodological point of view, neuropsychological and behavioral evaluations differ in several aspects: a) neuropsychological evaluations measure deficiencies during a test carried out at the laboratory, while everyday life evaluations refer to problems observed out of the laboratory, in particular at home or where patients spent most of their time; b) Neuropsychological evaluations mainly involve deterministic situations, each one having a single, intrinsic correct solution. On the contrary, daily life evaluations reflect the choices we make in order to resolve particular situations. Choices are not inherent in these situations. They are a complex interplay between the properties of the situations and our own properties, aspirations, and histories (Goldberg and Podell, 1999). Everyday life entails continuous changes in emotional states underlying a well known series of events, whereas neuropsychological evaluations involve restricted emotional states linked to the exposition to new events (i.e., anxiety) (Damasio, 1996). Thus, emotions could play a different role when solving cognitive problems and behavioral tasks.

Moreover, most cognitive tasks rely upon explicit mechanisms for understanding the task and for providing the response, while most of our actions in daily life are automatic or have little access to conscious monitoring (Lengfelder and Gollwitzer, 2001).

Studies on morality reflect this asymmetry. In the laboratory, moral judgment of normal humans seems to be mediated by moral reasoning, whereas in daily life, moral judgment generally is related to rapid, automatic evaluations (intuitions) (Haidt, 2001). Functional neuroimaging studies have shown that these two kinds of moral judgment are subserved by different brain regions (Moll et al., 2003).

In summary, neuropsychological and behavioral evaluations reflect different aspects of the organization of mind in prefrontal patients. The functional relationship between cognitive and behavioral abilities and the anatomical correlates subserving them still remain unclear, partly because of the lack of standard behavioral tests and the scarce number of multiple-case reports.

The recent development of structured questionnaires has allowed a more reliable assessment of patients with prefrontal lobe lesions, thus improving the identification and quantification of dysexecutive symptoms in everyday life (Godefroy et al., 2004; Wilson et al., 1996). However, to the best of our knowledge, no previous multiple-case studies using structured questionnaires to explore deficiencies in daily life in patients with lesions restricted to the prefrontal cortex have been reported. In addition, significant advances in neuroimaging techniques allow a more reliable characterization of focal cerebral lesions (i.e., a more precise definition of the topography and extension of lesions), directly improving the method of inferring function from human brain lesions (Rorden and Karnath, 2004).

In order to contribute to a better understanding of the role of prefrontal cortex in the control of complex human behavior, our study evaluates behavioral abilities in patients with prefrontal lesions using both structured questionnaires and modern neuroimaging methods. Our results suggest that patients with prefrontal lesions present a number of behavioral disturbances unrelated to cognitive deficiencies. In particular, lesions over the medial region as well as bilateral lesions are associated to greater behavioral troubles. Discussion of our results focuses on current models of the role of prefrontal cortex on behavioral and cognitive abilities.

\section{METHODS}

\section{Participants}

We studied 34 patients with focal prefrontal lesions and 40 healthy control participants. Frontal patients were 15 men and 19 women, 
mean age $48.0 \pm 12.5$ years and median educational level $10.5 \pm 3.7$ years. Age and years of education were not significantly different between men and women (age: Mann-Whitney test: $\mathrm{z}=1.48, \mathrm{p}=0.14$; years of education: Mann-Whitney test: $\mathrm{z}=-1.22$, $\mathrm{p}=0.12)$. Patients met the following inclusion criteria: i) presence of focal prefrontal lesion; ii) availability of Magnetic Resonance Imaging (MRI); iii) time since the lesion greater than 3 months. Patients were excluded if clinical examination demonstrated significant comprehension or expression deficit, severe aphasia, clinically detectable neglect, and psychiatric or neurological disorders. Etiology of the lesion corresponded to stroke $(n=14)$, excised benign primary tumor $(n=13)$ and trauma $(n=7)$. The main characteristics of patients are presented in Table 1 .

TABLE 1

Main characteristics of the patients

\begin{tabular}{|c|c|c|c|c|c|c|c|c|c|}
\hline Patient & Age & $\begin{array}{c}\text { Years } \\
\text { of }\end{array}$ & Gender & $\begin{array}{l}\text { Etiology } \\
\text { of lesion }\end{array}$ & $\begin{array}{c}\text { Frontal } \\
\text { Score }\end{array}$ & $\begin{array}{l}\text { ISDC }^{1} \\
\text { Score }\end{array}$ & $\begin{array}{l}\mathrm{DEX}^{2} \\
\text { Score }\end{array}$ & $\begin{array}{l}\text { Laterality } \\
\text { of the } \\
\text { lesion } \\
\text { in the } \\
\text { frontal } \\
\text { lobe }\end{array}$ & $\begin{array}{l}\text { Area of } \\
\text { of the } \\
\text { frontal } \\
\text { lobe } \\
\text { with } \\
\text { lesion }\end{array}$ \\
\hline $\mathrm{CSC}$ & 23 & 12 & M & Tumor & 56 & 24 & 34 & BIL & $\mathrm{im}^{* *}$ \\
\hline EMC & 26 & 12 & M & Trauma & 49 & 3 & 19 & LH & dl-sm \\
\hline EGM & 30 & 16 & M & Tumor & 81 & 1 & 7 & LH & sm-im \\
\hline GA & 34 & 11 & $\mathrm{~W}$ & Tumor & 74 & 40 & 29 & LH & $\mathrm{sm}$ \\
\hline MO & 36 & 6 & M & Cerebral trauma & 44 & 5 & 20 & LH & $\mathrm{im}$ \\
\hline $\mathrm{MN}$ & 36 & 8 & W & Tumor & 46 & 4 & 14 & $\mathbf{R H}$ & dl-sm-im \\
\hline LR & 37 & 9 & M & Vascular malformation & 63 & 25 & 28 & LH & $\mathrm{im}$ \\
\hline RGA & 38 & 11 & $\mathrm{M}$ & Cerebral trauma & 60 & 21 & 16 & BIL & sm-im \\
\hline MGL & 39 & 7 & W & Cerebral trauma & 63 & 50 & 27 & LH & $\mathrm{dl}$ \\
\hline MV & 39 & 16 & W & Tumor & 55 & 46 & 27 & LH & sm-im \\
\hline PTH & 39 & 8 & W & Tumor & 68 & 0 & 7 & RH & $\mathrm{im}$ \\
\hline MVO & 39 & 12 & W & Tumor & 83 & 8 & 27 & LH & dl-sm \\
\hline LV & 42 & 9 & M & Trauma & 60 & 14 & 9 & LH & $\mathrm{im}$ \\
\hline VAE & 43 & 12 & $\mathrm{~W}$ & Tumor & 45 & 62 & 34 & RH & $\mathrm{sm}$ \\
\hline VMR & 44 & 10 & M & Cerebral trauma & 63 & 74 & 59 & BIL & $\mathrm{im}$ \\
\hline JRR & 45 & 8 & M & Tumor & 58 & 27 & 33 & BIL & sm-im \\
\hline LD & 46 & 14 & W & Stroke & 61 & 42 & 13 & LH & $\mathrm{d} 1^{*}$ \\
\hline NRC & 48 & 8 & $\mathrm{~W}$ & Tumor & 73 & 3 & 25 & RH & $\mathrm{sm}$ \\
\hline HVG & 50 & 12 & M & Tumor & 56 & 32 & 31 & BIL & $\mathrm{sm} * * *$ \\
\hline MUM & 53 & 13 & W & Stroke & 61 & 5 & 9 & LH & $\mathrm{sm}$ \\
\hline EG & 53 & 3 & $\mathrm{~W}$ & Tumor & 61 & 20 & 24 & RH & dl-sm \\
\hline MRS & 54 & 4 & W & Stroke & 58 & 4 & 20 & RH & $\mathrm{dl}$ \\
\hline $\mathrm{MC}$ & 55 & 6 & W & Cerebral aneurysm & 66 & 6 & 4 & LH & dl-im \\
\hline EJSM & 55 & 6 & $\mathrm{~W}$ & Tumor & 50 & 35 & 19 & RH & $\mathrm{sm}$ \\
\hline EDG & 58 & 6 & W & Trauma & 73 & 0 & 22 & BIL & $\mathrm{im}$ \\
\hline JLV & 59 & 12 & M & Cerebral trauma & 77 & 10 & 20 & BIL & sm-im \\
\hline $\mathrm{ACU}$ & 60 & 12 & $\mathrm{~W}$ & Stroke & 76 & 7 & 7 & RH & $\mathrm{dl}$ \\
\hline ED & 60 & 1 & W & Stroke & 45 & 47 & 26 & LH & $\mathrm{sm}$ \\
\hline DSH & 60 & 12 & $\mathrm{~W}$ & Stoke & 62 & 109 & 38 & BIL & sm-im \\
\hline NIS & 62 & 12 & W & Stroke & 56 & 2 & 27 & BIL & $\mathrm{sm}$ \\
\hline GP & 63 & 5 & M & Vascular malformation & 48 & 96 & 50 & RH & sm-im \\
\hline $\mathrm{JCD}$ & 64 & 14 & M & Stroke & 57 & 0 & 15 & LH & $\mathrm{sm}$ \\
\hline EVM & 70 & 6 & $\mathrm{~W}$ & Stroke & 58 & 47 & 25 & BIL & dl-im \\
\hline AP & 73 & 14 & M & Cerebral trauma & 75 & 2 & 12 & LH & $\mathrm{im}$ \\
\hline
\end{tabular}

*: DL: Dorsolateral; **: IM: Inferomedial; ***: SM: Superomedial

1: ISDC: Inventaire du Syndrome Dysexécutif Comportemental

2: DEX : Dysexecutive questionnaire 
As control participants, we evaluated 16 men and 24 women; mean age $46.7 \pm 14.2$ years and educational level $10.3 \pm 3.1$ years. Control participants had no history of neurological or psychiatric disease, alcohol or drug abuse, or substance dependency. They presented a Mini-Mental State Examination (MMSE) (Folstein et al., 1975) score $\geq 25 / 30$, and they were matched to patients by age $[t(1,72)=$ $-0.41, \mathrm{p}=0.69]$ and by years of education $[t$ $(1,72)=-0.92, p=0.36]$.

Patients voluntarily agreed to participate in this study, signing an informed consent. This study was approved by the Ethics Committee of El Salvador Hospital, Santiago, Chile.

\section{Prefrontal function assessment}

In order to evaluate prefrontal functions of participants, neuropsychological and behavioral evaluations were applied. Neuropsychological tests were applied to patients and to a sample of control participants, matched by age, sex, and educational level, while behavioral questionnaires were applied only to patients and to caregivers or close relatives.

Individual scores in neuropsychological test as well as in behavioral evaluations were carried out in two to three consecutives sessions by a trained neuropsychologist (CP) blind to the anatomical distribution of the lesion in the patient group. All evaluations were done at the Neurology Service of El Salvador Hospital, Santiago, Chile, using standard material for neuropsychological tests.

\section{Neuropsychological assessment}

The neuropsychological assessment included the following tests:

a) Frontal Assessment Battery (FAB), a six sub-tests battery that evaluates the severity of the dysexecutive syndrome (Dubois et al., 2000).

b) Modified Version of the Wisconsin Card Sorting Test (MWCT), which evaluates conceptualization and cognitive flexibility. Performances in the MWCT were measured by scoring of the number of criteria achieved in the test as well as by scoring the number of preservatives errors (Nelson, 1976).

c) Verbal fluency was evaluated in three different tests: category verbal fluency (number of animal named by the participant in one minute); literal verbal fluency with letter $\mathrm{F}$ (number of words beginning with the letter $\mathrm{F}$ named by the participant in one minute); and literal verbal fluency with letter A number of words beginning with the letter A named by the participant in one minute). Literal fluency measured with two different letters provides a more reliable measure of spontaneous flexibility (Benton, 1968).

d) Luria's graphic series (1980), which provided a measure of motor and cognitive reactive flexibility.

e) Frontal behavior: prehension behavior, utilization of objects of the environment, and imitation of the examiner. This test evaluates the dependence of the participant on environmental cues and provides a measure of personal autonomy (Lhermitte et al., 1986). In addition, the scores of categories achieved in the MWCT, category and literal fluency, and Luria's graphic series were pooled to calculate a frontal score as a complementary measurement of the dysexecutive syndrome [Frontal score = (MWCT CRITERIA*3) + (category fluency + literal fluency with letter A + literal fluency with letter F) / 3 + Luria's graphic series]. Frontal score gives information about the severity of the cognitive deficit (Pillon et al., 1986).

\section{Behavioral assessment}

Behavioral abilities of patients were assessed using two structured questionnaires validated to evaluate key behavioral deficits secondary to prefrontal lesions:

i) The Dysexecutive questionnaire $(\mathrm{DEX})$ is a 20 -item questionnaire that prompts respondents to rate a variety of potential frontal functional problems using a five-point Likert scale (Burgess et al., 1998). Each item measures a particular 
aspect of behavioral disturbances. The rating of each single item is relatively independent of the rating of the others, and it could be analyzed independently. Each item takes the form of a brief statement. A higher rating indicates that the respondent perceives the problem as more frequent or severe. The items sample four broad areas of change or difficulty attributable to the dysexecutive syndrome, namely emotional or personality changes, motivational changes, behavioral changes, and cognitive changes (Stuss and Benson, 1984). The characteristics that each statement attempts to measure are functional problems impacting on everyday life. Due to frequent presence of anosognosia in patients with prefrontal lesions, the questionnaire is completed by a relative or a caregiver. The DEX also was completed by the patients themselves in order to calculate an anosognosia score (DEX completed by the relative minus DEX completed by the patient, a higher score indicating the presence of a more severe anosognosia). In addition, a global score in DEX obtained by simple addition of all items was computed. Global DEX score indicates the severity of behavioral disturbances in patients with prefrontal lesions considering each of the four areas of difficulty attributable to prefrontal lesions.

ii) "Inventaire du Syndrome Dysexécutif Comportemental" (ISDC): The ISDC questionnaire evaluates 12 dysexecutive behavior disturbances common in patients with prefrontal lesions: apathy; loss of planning of behavior; loss of interest; euphoria; irritability and aggressiveness; distractibility and hyperactivity; perseveration; loss of autonomy or dependence on social and physical environment; anosognosia (in this questionnaire, the presence and severity of anosognosia was evaluated by an informant); confabulation; social maladjustment; disturbances of sexual or alimentary behavior. Each item takes the form of a brief statement. The severity and frequency of dysexecutive behavior are rated on the basis of scripted questions administered to the patient's caregiver. Each behavior measurement is quoted on a scale between 0 to 3 for gravity and 0 to 4 for frequency. The score of each individual symptom is calculated by multiplying gravity score by frequency score. The rating of each behavioral disturbance is relatively independent from one another and it could be analyzed independently. In addition, a global ISDC score was calculated by adding all ratings. The global score provides information about the severity of behavioral disturbances (Godefroy et al., 2004).

\section{Neuroanatomical assessment}

Patients received a volumetric cerebral magnetic resonance imaging (MRI) scan in a Siemens $1.5 \mathrm{~T}$ scanner at Centro Radiológico Fleming, Santiago, Chile. Parameters were $T_{\mathrm{R}}=1500 \mathrm{~ms}, T_{\mathrm{E}}=2.03$ $\mathrm{ms}$, flip angle $=10^{\circ}$, matrix $(\mathrm{xyz})=$ $512 \times 512 \times 120$, slice thick $=1.5 \mathrm{~mm}$. A T1weighted volumetric MR sequence covered the entire brain (Frahm et al., 1986).

In order to identify the precise location of the lesion over the frontal lobe, the following steps were applied to the MRI image of each patient:

i) The lesion was drawn manually as a region of interest (ROI) on transversal slides by a researcher trained in medical neuroimaging processing who was blind to the results in neuropsychological evaluations. ROI creation procedure was performed using the software MRIcro (http: //www.psychology.nottingham.ac.uk/staff/ cr1/mricro.html) (Rorden and Brett 2000).

ii) Brain and lesion volumes were normalized using the software SPM (http: // www.fil.ion.ucl.ac.uk/spm/) following the procedure described in Brett et al. (2001).

iii) A $3 \mathrm{D}$ rendering of the normalized images was obtained using the software Anatomist (Rorden and Karnath, 2004) and MRIcro.

iv) The 3D image of the lesion was classified in anatomical categories based on its distribution over the hemispheres and over sub-areas of the frontal lobe. Standard hemispheric areas corresponded to left hemisphere $(\mathrm{LH})$, right hemisphere $(\mathrm{RH})$, and bilateral (BIL), while sub-areas of the frontal lobe corresponded to dorsolateral (DL), inferomedial (IM), and superomedial (SM) regions. The infero- and superomedial 
prefrontal cortex includes the orbital cortices, other mesial cortices and underlying white matter. This region also is called the ventromedial prefrontal cortex (Tranel and Damasio, 2002). Small lesions $(<10 \%$ of the total area) were ignored in the classification of patients.

\section{Statistical analysis}

In order to study neuropsychological and behavioral deficiencies in prefrontal patients, a non-parametric test was applied to the mean score of evaluations in the patient and in control groups. Patient/control comparisons allowed the study of the cognitive disturbances of prefrontal patients, whereas comparisons among patients allowed for the exploration of both cognitive and behavioral deficiencies associated with the damaged brain area.

\section{RESULTS}

\section{Comparisons between patient and control groups}

The score in neuropsychological evaluations of patient and control groups was submitting to a Mann-Whitney test with group as grouping variable.
The mean score in patients was significantly lower than in control participants in the following tests: category fluency (Mann-Whitney U: $\mathrm{z}=2.92, p=0.003$ ); lexical fluency with letter A (Mann-Whitney $\mathrm{U}: \mathrm{z}=2.11, p=0.034)$; Luria's graphic series (Mann-Whitney U: $\mathrm{z}=2.39, p=0.017$ ); number of criteria achieved in the MWCT (Mann-Whitney $\mathrm{U}: \mathrm{z}=2.6, p=0.007$ ); frontal behavior (Mann-Whitney $\mathrm{U}: \mathrm{z}=2.93$, $p=0.003$ ); and frontal score (Mann-Whitney $\mathrm{U}: \mathrm{z}=3.087, P=0.002)($ Table 2$)$.

\section{Comparisons within the patient group}

Table 3 shows the anatomical distribution of lesions. Due to the variability of lesions over the frontal lobe, some statistical comparisons were restricted to subgroups of patients (see Table 3 ).

In order to compare the anatomical correlation of neuropsychological and behavioral state, we restricted statistical comparison to four groups of patients: dorsolateral ( $D L, n=4)$, superomedial $(S M$, $\mathrm{n}=9$ ), and inferomedial (IM, $\mathrm{n}=8$ ) and to a group having lesions over both SM and IM (SM-IM, n=7) areas. For an example of each group, see Figure 1. SM-IM anatomical group was included as an independent group because medial lesions seem to develop common patterns of executive and behavioral deficit (Stuss et al., 2002).

TABLE 2

Mean scores in neuropsychological and behavioral evaluations in patient and control group

\begin{tabular}{lcccccccc}
\hline & \multicolumn{3}{c}{$\begin{array}{c}\text { Patient Group } \\
(\mathrm{n}=34)\end{array}$} & \multicolumn{3}{c}{$\begin{array}{c}\text { Control Group } \\
(\mathrm{n}=40)\end{array}$} & $\mathrm{p}$ \\
& Mean & Min & Max & Mean & Min & Max & \\
\hline Category Fluency & 16 & 7 & 29 & 20 & 10 & 28 & $\mathrm{p}=0.003$ \\
Fluency Letter F & 9 & 0 & 19 & 11 & 4 & 20 & $\mathrm{p}=0.034$ \\
Fluency Letter A & 9 & 0 & 16 & 11 & 3 & 20 & $\mathrm{p}=0.034$ \\
Luria's graphic series & 9 & 5 & 10 & 10 & 5 & 10 & $\mathrm{p}=0.017$ \\
number of criteria in the MWCT ${ }^{1}$ & 4 & 1 & 6 & 5 & 2 & 6 & $\mathrm{p}=0.007$ \\
Percentage of perseverative errors in the MWCT $^{2}$ & 6 & 0 & 15 & 5 & 0 & 20 & $\mathrm{p}>0.05$ \\
FAB & 14 & 4 & 18 & 15 & 10 & 18 & $\mathrm{p}>0.05$ \\
Frontal Behavior & 19 & 11 & 20 & 20 & 18 & 20 & $\mathrm{p}=0.003$ \\
Frontal Score & 33 & 16 & 49 & 40 & 25 & 50 & $\mathrm{p}=0.002$ \\
\hline
\end{tabular}

1: MWCT: Modified version of the Wisconsin Card Sorting Test

2: FAB: Frontal Assessment Battery 


\section{TABLE 3}

Distribution of the lesion by hemisphere and by region over the frontal lobe

\begin{tabular}{|c|c|c|c|c|}
\hline & Bilateral & Left Hemisphere Hemisphere $\mathrm{H}$ & Right Hemisphere & Total \\
\hline DL-IM ${ }^{1}$ & 1 & 1 & & 2 \\
\hline DL-SM-IM ${ }^{2}$ & & & 1 & 1 \\
\hline DL-SM ${ }^{3}$ & & 2 & 1 & 3 \\
\hline $\mathrm{DL}^{4}$ & & 2 & 2 & 4 \\
\hline $\mathrm{IM}^{5}$ & 3 & 4 & 1 & 8 \\
\hline SM-IM 6 & 4 & 2 & 1 & 7 \\
\hline $\mathrm{SM}^{7}$ & 2 & 4 & 3 & 9 \\
\hline Total & 10 & 15 & 9 & 34 \\
\hline
\end{tabular}

1: DL-IM: Dorsolateral-Inferomedial

2: DL-SM-IM: Dorsolateral-Superomedial and Inferomedial

3: DL-SM: Dorsolateral-Superomedial

4: DL: Dorsolateral

5: IM: Inferomedial

6: SM-IM: Supero- and Inferomedial

7: SM: Superomedial

Legend: Data in cells indicate the number of patients presenting a lesion in a particular combination of hemisphere and frontal lobe sub-region

Six patients presenting lesions over DL and medial areas were excluded from the analysis.

A Kruskal-Wallis test was applied to age and educational level with frontal lobe area as the grouping variable. No significant differences were observed (see Fig. 1).

\section{Neuropsychological evaluations by} anatomical areas

The score in neuropsychological evaluations within the patient group was subjected to a Kruskal-Wallis test (in several independent samples comparisons) and to a MannWhitney (in two independent samples comparisons) with frontal lobe area or hemisphere as grouping variables. No significant differences were found in any comparison (Table 4).

\section{Behavioral evaluations by anatomical areas}

A Kruskal-Wallis test was applied to the median score in global DEX, DEX items, global ISDC, and items ISDC, with frontal lobe area as the grouping variable (Table 4). A main effect of frontal lobe area was observed in confabulation $\left(\mathrm{X}^{2}(3)=8.51, \mathrm{P}\right.$ $<0.037)$.

When we restricted comparisons to pairs of frontal lobe areas, we found that the mean rank score in confabulation, restlessnesshyperkinesia, loss of planning of behavior, loss of interest, and anosognosia were significantly higher in SM-IM as compared to IM (Mann-Whitney U: $\mathrm{z}=2.38, \mathrm{P}<$ $0.017 ; \mathrm{z}=2.20, \mathrm{P}<0.027 ; \mathrm{z}=2.11, \mathrm{P}<$ $0.034 ; \mathrm{z}=1.98, \mathrm{P}<0.047$; and $\mathrm{z}=1.90, \mathrm{P}<$ 0.050 , respectively).

\section{Effects of hemisphere on behavior}

Due to the restricted number of patients, the effect of hemisphere was measured comparing three groups of patients, regardless of the frontal lobe lesion area. The three groups were: a) patients with lesions over the LH ( $\mathrm{LH}, \mathrm{n}=12)$; b) patients with lesions over the $\mathrm{RH}(\mathrm{RH}, \mathrm{n}=7)$; and $\mathrm{c})$ patients with bilateral lesions (BIL, $n=9)$. A Kruskal-Wallis test was applied to the scores in neuropsychological and behavioral evaluations with hemisphere ( $\mathrm{LH}, \mathrm{RH}$, and BIL) as the grouping variable. A main effect of hemisphere was observed in confabulation 


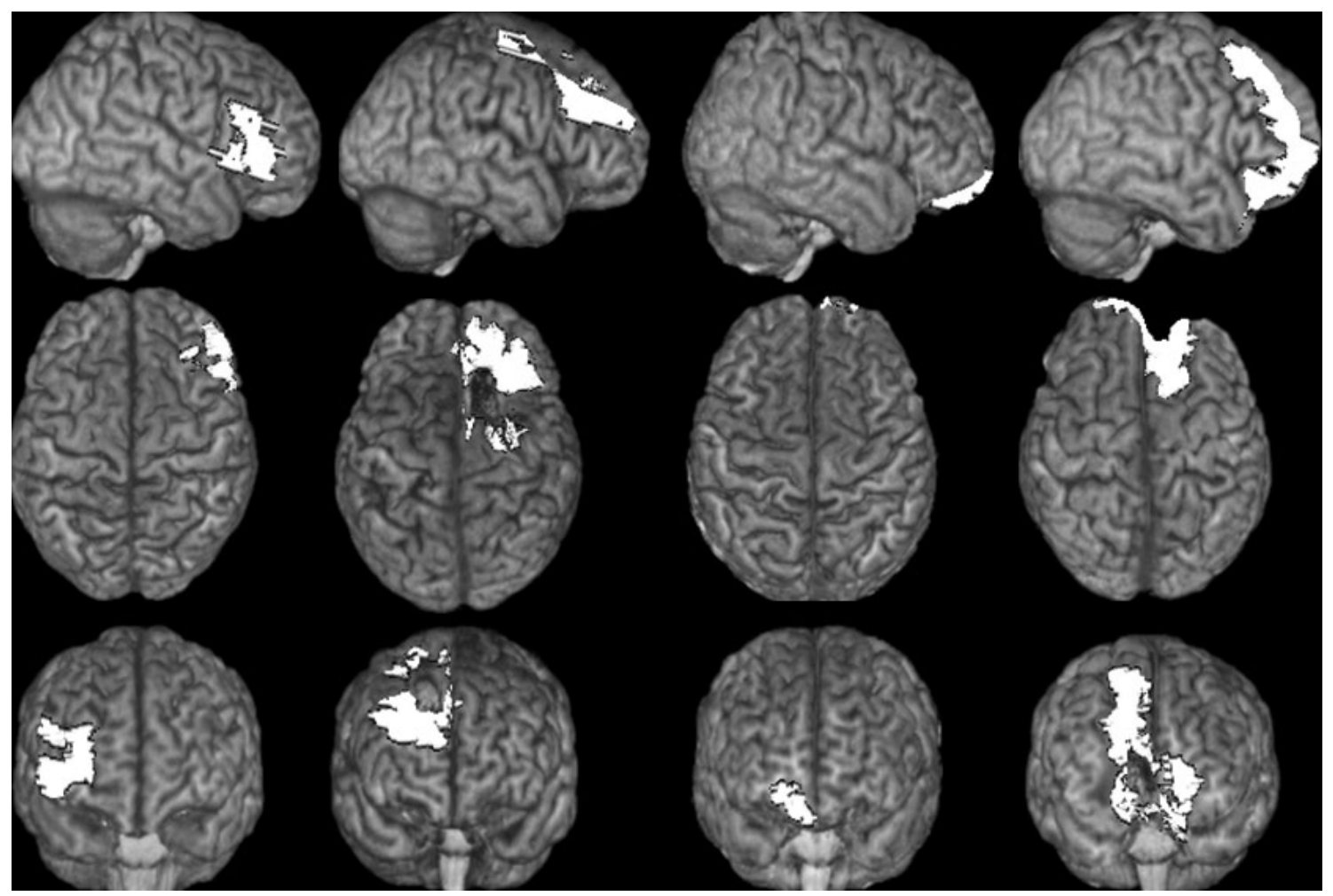

FIGURE 1: Brain 3D image reconstruction in four patients.

Legend: First row shows saggital, second row axial and third row coronal view of the lesion in the same patient. First column illustrates a DL lesion, second column a SM lesion, third column a IM lesion and fourth column a SM-IM lesion

$\left(\mathrm{X}^{2}(2)=5.87, P<0.05\right)$. When we restricted comparisons to pairs of hemispheric groups, we found that the mean score in planning problems, lack of insight and social awareness, shallowing of affective response, poor decision-making ability, no concern for social rules, and in global DEX were significantly higher in BIL lesions as compared to $\mathrm{LH}$ (Mann-Whitney $\mathrm{U}: \mathrm{z}=$ $2.53, P<0.011 ; \mathrm{z}=2.72, P<0.006 ; \mathrm{z}=$ $2.50, P<0.012 ; \mathrm{z}=2.21, P<0.027 ; \mathrm{z}=$ $3.53, P<0.001 ; \mathrm{z}=2.23, P<0.026$; and $\mathrm{z}=$ $2.50, P<0.012$; respectively).

In addition, the mean score in planning problems and poor decision-making ability was significantly higher in BIL lesions as compared to $\mathrm{RH}$ lesions (Mann-Whitney U: $\mathrm{z}=2.46, P<0.014 ;$ and $\mathrm{z}=2.02, P<$ 0.042 ; respectively). No differences between $\mathrm{LH}$ and $\mathrm{RH}$ were found.
In summary, the main results of our study are: i) confabulation, restlessnesshyperkinesia, impaired planning, loss of interest and anosognosia were more frequent in patients with SM-IM as compared to IM lesions; ii) planning problems, lack of insight and social awareness, shallowing of affective response, poor-decision making ability, no concern for social rules, and in global DEX were significantly higher in BIL lesions as compared to LH; iii) planning problems and poor decision-making ability were significantly higher in BIL lesions as compared to RH; iv) behavioral problems were not related to cognitive problems, i.e., we did not find significant differences related to lesion location on cognitive measurement, while we did find them in several behavioral evaluations. 
TABLE 4

Mean scores in neuropsychological and behavioral evaluations by damaged areas of the frontal lobe

\begin{tabular}{|c|c|c|c|c|c|c|c|c|c|c|c|c|}
\hline & \multicolumn{3}{|c|}{$\mathrm{DL} *(\mathrm{n}=4)$} & \multicolumn{3}{|c|}{$\mathrm{IM}^{* *}(\mathrm{n}=8)$} & \multicolumn{3}{|c|}{ SM-IM*** $(\mathrm{n}=7)$} & \multicolumn{3}{|c|}{$\mathrm{SM}^{* * * *}(\mathrm{n}=9)$} \\
\hline & Mean & Min & $\operatorname{Max}$ & Mean & Min & $\operatorname{Max}$ & Mean & Min & $\operatorname{Max}$ & Mean & Min & $\operatorname{Max}$ \\
\hline Category Fluency & 17 & 9 & 27 & 16 & 8 & 22 & 18 & 10 & 29 & 16 & 7 & 24 \\
\hline Fluency Letter F & 10 & 4 & 16 & 10 & 1 & 19 & 11 & 0 & 19 & 8 & 0 & 19 \\
\hline Fluency Letter A & 9 & 4 & 13 & 10 & 3 & 16 & 9 & 2 & 16 & 7 & 0 & 13 \\
\hline Luria's graphic series & 10 & 10 & 10 & 10 & 9 & 10 & 9 & 7 & 10 & 9 & 5 & 10 \\
\hline $\begin{array}{l}\text { MWCT }^{1} \# \text { criteria } \\
\text { MWCT \% }\end{array}$ & 4 & 2 & 6 & 5 & 4 & 6 & 4 & 2 & 6 & 5 & 2 & 6 \\
\hline perseveration error & 5 & 0 & 14 & 5 & 1 & 13 & 5 & 0 & 13 & 5 & 0 & 14 \\
\hline $\mathrm{FAB}^{2}$ & 13 & 10 & 17 & 15 & 12. & 18 & 15 & 11 & 18 & 12 & 4 & 17 \\
\hline Frontal Behaviors & 18 & 16 & 20 & 20 & 20 & 20 & 17 & 13 & 20 & 18 & 11 & 20 \\
\hline Frontal Score & 35 & 22 & 47 & 37 & 27 & 46 & 35 & 19 & 49 & 34 & 21 & 47 \\
\hline ISDC $^{3}$ Global & 26 & 4 & 50 & 18 & 0 & 74 & 44 & 1 & 109 & 25 & 0 & 62 \\
\hline Apathy & 5 & 2 & 9 & 5 & 0 & 12 & 7 & 0 & 12 & 4 & 0 & 9 \\
\hline \multicolumn{13}{|l|}{ Loss of Behavior } \\
\hline Planning & 5 & 0 & 9 & 2 & 0 & 6 & 6 & 0 & 12 & 3 & 0 & 8 \\
\hline Loss of Interest & 3 & 0 & 6 & 2 & 0 & 8 & 6 & 0 & 12 & 3 & 0 & 8 \\
\hline Euphoria & 0 & 0 & 0 & 2 & 0 & 12 & 2 & 0 & 6 & 1 & 0 & 4 \\
\hline \multicolumn{13}{|l|}{ Irritability and } \\
\hline Aggressiveness & 2 & 0 & 4 & 2 & 0 & 12 & 4 & 0 & 12 & 2 & 0 & 6 \\
\hline \multicolumn{13}{|l|}{ Distractibility and } \\
\hline Hyperactivity & 3 & 0 & 9 & 2 & 0 & 12 & 4 & 0 & 12 & 2 & 0 & 6 \\
\hline Perseveration & 2 & 0 & 6 & 1 & 0 & 6 & 3 & 0 & 12 & 2 & 0 & 8 \\
\hline \multicolumn{13}{|l|}{ Loss of Autonomy or } \\
\hline \multicolumn{13}{|l|}{$\begin{array}{l}\text { Dependence on Social } \\
\text { and Physical }\end{array}$} \\
\hline Environment & 1 & 0 & 4 & 1 & 0 & 6 & 2 & 0 & 9 & 3 & 0 & 9 \\
\hline ISDC Anosognosia & 1 & 0 & 4 & 0 & 0 & 0 & 2 & 0 & 6 & 2 & 0 & 12 \\
\hline Confabulation & 3 & 0 & 9 & 0 & 0 & 0 & 2 & 0 & 6 & 0 & 0 & 2 \\
\hline Social Maladjustment & 1 & 0 & 4 & 1 & 0 & 6 & 3 & 0 & 12 & 0 & 0 & 2 \\
\hline \multicolumn{13}{|l|}{ Sexual and Alimentary } \\
\hline Behavior Problems & 1 & 0 & 2 & 1 & 0 & 2 & 4 & 0 & 12 & 2 & 0 & 8 \\
\hline Global DEX ${ }^{4}$ & 17 & 7 & 27 & 24 & 7 & 59 & 27 & 7 & 50 & 24 & 9 & 34 \\
\hline \multicolumn{13}{|l|}{ Abstract thinking } \\
\hline problems & 2 & 0 & 2 & 1 & 0 & 4 & 1 & 0 & 2 & 1 & 0 & 3 \\
\hline Impulsivity & 1 & 0 & 1 & 2 & 0 & 4 & 1 & 0 & 3 & 1 & 0 & 2 \\
\hline Confabulation & 0 & 0 & 1 & 0 & 0 & 2 & 0 & 0 & 2 & 0 & 0 & 0 \\
\hline Planning problems & 1 & 0 & 4 & 2 & 0 & 4 & 1 & 0 & 4 & 1 & 0 & 2 \\
\hline Euphoria & 1 & 0 & 2 & 1 & 0 & 3 & 1 & 0 & 3 & 1 & 0 & 2 \\
\hline \multicolumn{13}{|l|}{ Temporal sequencing } \\
\hline deficits & 1 & 0 & 2 & 1 & 0 & 4 & 1 & 0 & 3 & 1 & 0 & 2 \\
\hline \multicolumn{13}{|l|}{ Lack of insight and } \\
\hline social awareness & 1 & 0 & 2 & 1 & 0 & 3 & 2 & 0 & 4 & 2 & 0 & 3 \\
\hline Apathy and lack of drive & 2 & 1 & 4 & 2 & 0 & 4 & 2 & 0 & 4 & 2 & 0 & 4 \\
\hline Disinhibition & 1 & 0 & 2 & 2 & 0 & 4 & 1 & 0 & 2 & 1 & 0 & 2 \\
\hline Variable motivation & 1 & 0 & 2 & 2 & 0 & 4 & 1 & 0 & 2 & 1 & 0 & 2 \\
\hline
\end{tabular}


Cont. Table 4.

\begin{tabular}{|c|c|c|c|c|c|c|c|c|c|c|c|c|}
\hline & \multicolumn{3}{|c|}{$\mathrm{DL}^{*}(\mathrm{n}=4)$} & \multicolumn{3}{|c|}{$\mathrm{IM}^{* *}(\mathrm{n}=8)$} & \multicolumn{3}{|c|}{ SM-IM*** $(\mathrm{n}=7)$} & \multicolumn{3}{|c|}{$\mathrm{SM} * * * *(\mathrm{n}=9)$} \\
\hline & Mean & Min & $\operatorname{Max}$ & Mean & Min & Max & Mean & Min & Max & Mean & Min & Max \\
\hline \multicolumn{13}{|l|}{ Swallowing of } \\
\hline affective response & 1 & 0 & 2 & 1 & 0 & 4 & 2 & 0 & 4 & 2 & 0 & 3 \\
\hline Aggression & 1 & 0 & 2 & 1 & 0 & 4 & 2 & 1 & 4 & 1 & 0 & 2 \\
\hline Lack of concern & 0 & 0 & 1 & 1 & 0 & 3 & 1 & 0 & 4 & 1 & 0 & 2 \\
\hline Perseveration & 1 & 0 & 2 & 1 & 0 & 3 & 1 & 0 & 4 & 1 & 0 & 3 \\
\hline Restlessness- & & & & & & & & & & & & \\
\hline Hyperkinesia & 1 & 0 & 2 & 1 & 0 & 4 & 2 & 0 & 3 & 1 & 0 & 2 \\
\hline Inability to inhibit & & & & & & & & & & & & \\
\hline response & 1 & 0 & 2 & 1 & 0 & 4 & 0 & 0 & 1 & 1 & 0 & 3 \\
\hline Knowing-doing & & & & & & & & & & & & \\
\hline dissociation & 1 & 0 & 2 & 1 & 0 & 4 & 1 & 0 & 3 & 1 & 0 & 4 \\
\hline Distractibility & 2 & 0 & 3 & 1 & 0 & 3 & 2 & 0 & 3 & 2 & 0 & 3 \\
\hline $\begin{array}{l}\text { Poor decision-making } \\
\text { ability }\end{array}$ & 1 & 0 & 2 & 2 & 0 & 4 & 3 & 0 & 4 & 1 & 0 & 3 \\
\hline $\begin{array}{l}\text { No concern for } \\
\text { social rules }\end{array}$ & 1 & 0 & 2 & 2 & 0 & 4 & 2 & 0 & 4 & 1 & 0 & 4 \\
\hline DEX Anosognosia & -9 & -35 & 13 & -2 & -25 & 56 & 1 & -36 & 33 & -9 & -28 & 10 \\
\hline
\end{tabular}

*: DL: Dorsolateral; **: IM: Inferomedial; ***; SM-IM: Supero- and Inferomedial;

$* * * *$ : SM: Superomedial

1: MWCT: Modified version of the Wisconsin Sorting Test

2: FAB: Frontal Assessment Battery

3: ISDC: Inventaire du Syndrome Dysexécutif Comportemental

4: DEX: Dysexecutive questionnaire

\section{DISCUSSION}

Many studies have established a connection between lesions in the prefrontal lobe and cognitive and behavioral disturbances (for a review, see Damasio and Anderson, 2003). However, the relationship between clinical abnormalities of behavior and cognitive deficits observed on formal testing has been examined in only a few studies. Until the advent of structured questionnaires aimed at assessing behavior in daily life, most of the significant data came from the evaluations of single-case patients at the laboratory, limiting the access to important aspects of the behavioral state of patients that are expressed only in unstructured environments as at home (Godefroy, 2003). In order to contribute to a better understanding of the role of prefrontal cortex in the control of complex human behavior, we have assessed a series of patients with strictly prefrontal lesions, using cognitive tests sensitive to prefrontal dysfunction, structured questionnaires sensible to behavioral disturbances in daily life and modern neuroimage methods.

Before trying to explain our principal results, we believe it is important to address some criticisms that can be made of our study. Two main objections to the validity of our results can be formulated. The first objection made be made with regard to the small number of patients included in the study. Indeed, patients with strictly limited prefrontal lesions are infrequent, and the recruitment of the thirty-four patients took over two years. In particular, the DL group contained a very low number of patients. However, the application of nonparametrical tests allowed us to reduce statistical errors due to the small number of subjects (Motulsky, 1995). The second objection would indicate the heterogeneity of the etiology of the lesions. However, previous studies have shown that 
performances in cognitive tasks of patients with focal prefrontal lesions secondary to infarctions and to excised meningioma or benign gliomas did not differ (Stuss et al., 2002). Nevertheless, in order to support our present results, we will take into account both the number of patients and etiology variables in future studies.

Our results support current evidence about the impact of prefrontal lesions on the cognitive state of patients. In almost all neuropsychological evaluations, patients exhibit cognitive deficiencies as compared to the matched control group. Nevertheless, we did not find any correlation between lesions in a particular prefrontal region and poor performance in cognitive test. In composite cognitive tasks, such as the Wisconsin card-sorting test, the Stroop test, lexical fluency, or the graphic series, several cortical networks are actuated, including prefrontal, cingulated, parietal and occipital cortices (Stuss et al., 2002). For example, superior medial frontal damage, right or left, and dorsolateral frontal lesions resulted in impairment in letter-based fluency performance (Stuss et al., 1998). Cognitive deficiencies assessed in these tests rely on frontal lobe abilities, such as shifting (shifting back and forth between multiple cognitive tasks or mental set), monitoring information for relevance to the task at hand, and appropriately updating the information content with newer, relevant information and inhibiting dominant, automatic or preponderant information (Miyake et al., 2000). Thus, the above-mentioned series of patients with prefrontal lesions performed more poorly than controls in particular cognitive tasks. In summary, our results show a global deficiency, but we found no correlation between specific deficits and anatomical areas, probably because of the small number of patients with dorsolateral lesions included in the study and/or the multiple composite characteristics of our neuropsychological evaluation, i.e. performance on each task relied on different cognitive processes subserved by the prefrontal cortex (switching, monitoring, etc.) and by retrorolandic cortex (language, visual recognition, etc.)
Our results comparing prefrontal lobe areas support the idea that simultaneous combination of superior and inferior medial lesions are associated with severe behavioral disturbance as compared with isolated IM lesion. In fact, indifference to social rules and a marked personality change often constitute the most striking symptoms in patients with bilateral orbitofrontal (ventromedial) pathology (Blair and Cipolotti, 2000; Mataro et al., 2001). Most single-case reports of patients with prefrontal lesions and severe behavioral disturbances presented lesions in SM and IM prefrontal regions. Damasio et al. (1994) have demonstrated that Phineas Gage's lesions involved the ventromedial region of the prefrontal cortex bilaterally, including mesial sectors of areas 10 and 11 , areas 12, 13 and 25, and subgenual sectors of areas 24 and 32 of Brodmann. Dimitrov et al. (1999) described patient MGS who presented diminished sensitivity to socially relevant stimuli and situational nuances, impairment in sexual behavior, lost of sense of responsibility, and disinhibition. MGS' lesion also involved SM-IM frontal regions (Brodmann's areas 8, 9, 10, 11, 24 and 32).

IM prefrontal circuitry provides a connection between regions engaged in emotions (Brodmann's areas 11, 13, 14) and motivation (Brodmann's areas 25, 32) Moreover, bearing in mind the connections between the orbitofrontal cortex and the limbic system, a key role in the modulatory influence of the frontal lobes on limbic activity may be assumed. As stated by Nauta (1971): "the failures of the affective and motivational responses of the frontal patient to match environmental situations that he nonetheless can describe accurately could thus be tentatively interpreted as the consequence of a loss of a modulatory influence normally exerted by the neocortex on the limbic mechanism of the frontal lobe."

The role of SM cortex in attention could play a role in the higher severity of the behavioral deficit. SM areas (Brodmann's area 8) subtend attentional circuitry involved in sustained attention and attentional switching, two very important functions in daily life routine (Sanides 
1972). Moreover, SM prefrontal region also are involved in activation, initiation and maintenance, processes intervening in daily life activities.

To some extent, our results support the idea that behavioral abilities could have a wide representation over both IM and SM areas of the frontal lobe. Thus, in the case of isolated IM or SM damage, behavioral abilities relying mostly on one of the above-mentioned areas could be carried out partially by the remaining normal medial areas.

Previous studies in patients with ventromedial lesions have demonstrated disturbances in decision-making capacity that could account for behavioral disturbances of these patients (Bechara et al., 1994; 1997). Nevertheless, the role of local circuits over medial areas must be explored in future studies focusing on specific dimensions of behavior.

Behavior disturbances are more frequent when patients present bilateral prefrontal lesions, as has been found in a previous study: the scores on behavioral scales assessing planning abilities in patients with prefrontal lesions were correlated mainly with hipometabolism in bilateral orbitofrontal cortex (Sarazin et al., 1998).

Our results suggest that behavioral abilities could rely at least in part on bilaterally represented processes. Thus, when lesions are restricted to one hemisphere, the subtended functions could be carried out by the remaining non-injured hemisphere.

Our results regarding the association between behavioral disturbances and lesions in the prefrontal lobe area are particularly relevant considering that the cognitive disturbances in patients with lesions in different prefrontal areas were not significantly different. This result suggests a dissociation between cognition and behavior in patients with prefrontal lesions. As has been suggested by Burgess et al. (1998), it is perfectly plausible that the situation of a person performing a cognitive task subserved by the prefrontal cortex under strict examination conditions might be different from most situations in the real world. Thus, there is little correspondence between the cognitive resources required in the examination conditions and those required in real-world situations. Certainty of the implicit approaches adopted in the design of neuropsychological tests make the patient's impairments less likely to become manifest in the test situation. In neuropsychological tests, the patient typically has a single explicit problem to tackle at any one time, the trials tend to be very short, task initiation is strongly prompted by the examiner, and what constitutes successful trial completion is clearly characterized. Patients are rarely required to organize or plan their behavior over longer periods of time or to set priorities when confronted with two or more competing tasks. Yet these kinds of "executive" abilities are a large component of everyday activities (Shallice and Burgess, 1991).

Nevertheless, the modular organization of prefrontal cortex suggested by the literature, i.e., that behavior troubles are mainly secondary to ventromedial lesions and not necessary associated with poor performances in cognitive tasks, are probably not explained only by the facts described above (Sarazin et al., 1998). A defect in the activation of somatic markers that must accompany the internal and automatic processing of possible response options, interacting with the premorbid personality and the solicitation of environment, may provoke either indecision and inactivity or lack of concern about the consequences of action and disinhibited activity (Bechara et al., 1997; 2000). Our data are in agreement with such a regulating role of ventromedial prefrontal cortex in behavior.

The main conclusions of this first multiple-case study, which applies structured questionnaire to explore the deficiencies in daily life in patients with lesions restricted to the prefrontal cortex, are: 1) that severe behavior problems in daily life are preferentially associated with lesions involving medial prefrontal cortex, i.e., simultaneous damage over SM and IM regions; and 2) that there is a dissociation between behavioral disturbances in daily life and problems in cognitive tasks 
performed in the laboratory. Local superoand inferomedial circuits and a bilateral representation of behavioral evaluation are proposed. Future studies certainly will contribute to clarify the role of prefrontal lobe in behavioral control and its relationship to cognitive abilities in humans.

\section{ACKNOWLEDGEMENTS}

Supported by FONDECYT Project \#1020333. We are indebted to the patients and their families who voluntarily agreed to participate in this study. We thank Mauricio Farías for his valuable collaboration in performing MRIs.

\section{REFERENCES}

BECHARA A, DAMASIO AR, DAMASIO H, ANDERSON SW (1994) Insensitivity to future consequences following damage to human prefrontal cortex. Cognition 50: 7-15

BECHARA A, DAMASIO H, TRANEL D, DAMASIO AR (1997) Deciding advantageously before knowing the advantageous strategy [see comments]. Science 275: 1293-1295

BECHARA A, TRANEL D, DAMASIO H (2000) Characterization of the decision-making deficit of patients with ventromedial prefrontal cortex lesions. Brain 123: 2189-2202

BENTON A (1968) Differential behavior effects in frontal lobe disease. Neuropsychologia 6: 53-60

BLAIR RJ, CIPOLOTTI L (2000) Impaired social response reversal. A case of "acquired sociopathy." Brain 123: $1122-1141$

BRETT M, LEFF AP, RORDEN C, ASHBURNER J (2001) Spatial normalization of brain images with focal lesions using cost function masking. Neuroimage 14: 486-500

BURGESS PW, ALDERMAN N, EVANS J, EMSLIE H, WILSON BA (1998) The ecological validity of tests of executive function. J Int Neuropsychol Soc 4: 547-558

CHAN RC (2001) Dysexecutive symptoms among a nonclinical sample: A study with the use of the Dysexecutive Questionnaire. Br J Psychol 92 Part 3: 551-565

DAMASIO AR (1996) The somatic marker hypothesis and the possible functions of the prefrontal cortex PhilosTrans R Soc Lond B Biol Sci 351: 1413-1420

DAMASIO AR, ANDERSON SW (2003) The Frontal Lobes. In: HEILMAN KM, VALENSTEIN E (eds) Clinical Neuropsychology. Oxford: Oxford University Press. pp: 404-446

DAMASIO H, GRABOWSKI T, FRANK $\mathrm{R}$, GALABURDA AM, DAMASIO AR (1994) The return of Phineas Gage: Clues about the brain from the skull of a famous patient. Science 264: 1102-1105

DIMITROV M, PHIPPS M, ZAHN TP, GRAFMAN J (1999) A thoroughly modern Gage. Neurocase 5: 345354
DUBOIS B, SLACHEVSKY A, LITVAN I, PILLON B (2000) The FAB: A Frontal Assessment Battery at bedside. Neurology 55: 1621-1626

ESLINGER PJ, DAMASIO AR (1985) Severe disturbance of higher cognition after bilateral frontal lobe ablation: Patient EVR. Neurology 35: 1731-1741

FOLSTEIN MF, FOLSTEIN SE, MCHUGH PR (1975) "Mini-mental state". A practical method for grading the cognitive state of patients for the clinician. Journal of Psychiatry Research 12: 189-198

FRAHM J, HAASE A, MATTHAEI D (1986) Rapid threedimensional MR imaging using the FLASH technique. J Comput Assist Tomogr 10: 363-368

GODEFROY O (2003) Frontal syndrome and disorders of executive functions. J Neurol 250: 1-6

GODEFROY O, BRIGITTE A, PHILIPPE A, MIRA DH, OLIVIER G, DIDIER LE $G$, ROSE-MARIE M, THIERRY M, CHRYSTELE M, BLANDINE $P$, BERNARD P, PHILIPPE R (2004) [Frontal dysexecutive syndromes]. Rev Neurol (Paris) 160: 899-909

GOLDBERG E, PODELL K (1999) Adaptive versus veridical decision making and the frontal lobes. Conscious Cogn 8: 364-377

HAIDT J (2001) The emotional dog and its rational tail: A social intuitionist approach to moral judgment. Psychol Rev 108: 814-834

LENGFELDER A, GOLLWITZER PM (2001) Reflective and reflexive action control in patients with frontal brain lesions. Neuropsychology 15: 80-100

LHERMITTE F, PILLON B, SERDARU M (1986) Human autonomy and the frontal lobes. Part I: Imitation and utilization behavior: a neuropsychological study of 75 patients. Ann Neurol 19: 326-334

LURIA A (1980) Higher Cortical Functions in Man. New York: NY: Basic Books Inc Publishers

MACMILLAN M (1996) Phineas Gage: A case for all reasons. In: CODE C, WALLESCH C, ROCH LECOURS A (eds) Classic cases in neuropsychology. Hove: Psychology Press. pp: 243-262

MATARO M, JURADO MA, GARCIA-SANCHEZ C, BARRAQUER L, COSTA-JUSSA FR, JUNQUE C (2001) Long-term effects of bilateral frontal brain lesion: 60 years after injury with an iron bar. Arch Neurol 58: 1139-1142

MIYAKE A, EMERSON MJ, FRIEDMAN NP (2000. Assessment of executive functions in clinical settings: Problems and recommendations. Semin Speech Lang 21: $169-183$

MOLL J, DE OLIVEIRA-SOUZA R, ESLINGER PJ (2003) Morals and the human brain: A working model. Neuroreport 14: 299-305

MOTULSKY H (1995) Intuitive Biostatistics. New York: Oxford University Press

NAUTA WJ (1971) The problem of the frontal lobe: A reinterpretation. J Psychiatr Res 8: 167-187

NELSON HE (1976). A modified card sorting test sensitive to frontal lobe defects. Cortex 12: 313-324

PILLON B, DUBOIS B, LHERMITTE F, AGID Y (1986) Heterogeneity of cognitive impairment in progressive supranuclear palsy, Parkinson's disease, and Alzheimer's disease. Neurology 36: 1179-1185

RORDEN C, BRETT M (2000) Stereotaxic display of brain lesions. Behav Neurol 12: 191-200

RORDEN C, KARNATH HO (2004) Using human brain lesions to infer function: A relic from a past era in the fMRI age? Nat Rev Neurosci 5: 813-819

SANIDES (1972) Representation in the cerebral cortex and its areal laminar pattern. In: BOURNE G (eds) Structure and function of the nervous tissue. New York: Academic Press. vol. 5. pp: 329-423 
SARAZIN M, PILLON B, GIANNAKOPOULOS P, RANCUREL G, SAMSON Y, DUBOIS B (1998) Clinico-metabolic dissociation of cognitive functions and social behavior in frontal lobe lesions. Neurology 51: $142-148$

SHALLICE T, BURGESS PW (1991) Deficits in strategy application following frontal lobe damage in man. Brain 114: 727-741

STUSS DT, ALEXANDER MP, FLODEN D, BINNS M, LEVINE B, MCINTOSH AR, RAJAH N, HEVENOR S (2002) Fractionation and localization of distinct frontal lobe processes: Evidence from focal lesions in humans. In: STUSS DT, KNIGHT RT (eds) Principles of Frontal Lobe Function. Oxford: Oxford University Press. pp: 392-408
STUSS DT, ALEXANDER MP, HAMER L, PALUMBO C, DEMPSTER R, BINNS M, LEVINE B, IZUKAWA D (1998) The effects of focal anterior and posterior brain lesions on verbal fluency. J Int Neuropsychol Soc 4: 265-278

STUSS DT, BENSON DF (1984) Neuropsychological studies of the frontal lobes. Psychol Bull 95: 3-28

TRANEL D, DAMASIO AR (2002) The neuropsychologic consequences of frontal lobes lesions. In: GRAFMAN J (ed) Handbook of neuropsychology. Amsterdam: Elsevier. vol. 7. pp: 145-156

WILSON B, ALDERMAN N, BURGESS P, EMSLIE H, EVANS JJ (1996) Behavioral Assessment of the Dysexecutive Syndrome (BADS). Bury St Edmunds: Thames Valley Test Company 